\title{
Retraction Note to: A Negotiation Method for Task Allocation with Time Constraints in Open Grid Environments
}

Yan Kong, Minjie Zhang, Dayong Ye and Xudong Luo

Retraction Note to:

Chapter "A Negotiation Method for Task Allocation with Time Constraints in Open Grid Environments" in: K. Fujita et al. (eds.), Next Frontier in Agent-Based Complex Automated Negotiation, Studies in Computational Intelligence 596, https://doi.org/10.1007/978-4-431-55525-4_2

The editors have retracted this chapter [1] because of significant overlap with a previously published article by the same authors [2]. Authors Yan Kong, Minjie Zhang and Dayong Ye agree with this retraction. Author Xudong Luo could not be reached to comment on this retraction.

[1] Yan Kong, Minjie Zhang, Dayong Ye and Xudong Luo. A Negotiation Method for Task Allocation with Time Constraints in Open Grid Environments. In K. Fujita et al. (eds.), Next Frontier in Agent-Based Complex Automated Negotiation, Studies in Computational Intelligence 596, DOI 10.1007/ 978-4-431-55525-4_2.

[2] Yan Kong, Minjie Zhang and Dayong Ye. "A Negotiation-based Method for Task Allocation with Time Constraints in Open Grid Environments". CONCURRENCY AND COMPUTATION: PRACTICE AND EXPERIENCE. Concurrency Computat.: Pract. Exper. (2014) Published online in Wiley Online Library (wileyonlinelibrary.com). DOI: 10.1002/cpe.3271.

The retracted online version of this chapter can be found at https://doi.org/10.1007/978-4-431-55525-4_2 